\title{
A Note on Burkholder Integrals
}

\author{
Xiaogao Feng ${ }^{1,2, *}$ and Shengjin $\mathrm{Huo}^{3}$ \\ ${ }^{1}$ College of Mathematics and Information, China West Normal University, \\ Nanchong 637002, P.R. China. \\ 2 Department of Mathematics, Soochow University, Suzhou 215006, P.R. China. \\ ${ }^{3}$ Department of Mathematics, Tianjin Polytechnic University, TianJin 300387, \\ P.R. China.
}

Received 11 April 2015; Accepted (in second revised version) 9 December 2015

\begin{abstract}
In this note, for $k$-quasiconformal mappings of a bounded domain into the complex plane, we give an upper bound of Burkholder integral. Moreover, as an application we obtain an upper bound of the $L^{p}$-integral of $\sqrt{J_{f}}$ and $|D f|$ for certain $K$-quasiconformal mappings.
\end{abstract}

AMS subject classifications: 30C62,30A10

Key words: Quasiconformal mapping, Burkholder function, Morrey's problem.

\section{Introduction}

Let $\Omega$ and $\Omega^{\prime}$ be two bounded simply connected domains of the complex plane $\mathbb{C}$. A homeomorphism $f: \Omega \longrightarrow \Omega^{\prime}$ is called $k$-quasiconformal, if it belongs locally to the Sobolev class $\mathbb{W}_{\text {loc }}^{1,2}(\Omega)$ and satisfies the Beltrami equation

$$
\frac{\partial f}{\bar{\partial} z}=\mu_{f} \frac{\partial f}{\partial z} \quad \text { a.e. } \quad z \in \Omega,
$$

where the Beltrami coefficient has bounded $L_{\infty}$ norm: $\left\|\mu_{f}\right\|_{\infty} \leq k<1$. In particular, a homeomorphism of $\mathbb{C}$ onto itself is called principal solution of the Beltrami equation

$$
\frac{\partial f}{\bar{\partial} z}=\mu_{f} \frac{\partial f}{\partial z}
$$

if it satisfies the asymptotical normalization condition

$$
f(z)=z+\frac{b_{1}}{z}+\frac{b_{2}}{z^{2}}+\cdots, \quad \text { for } \quad|z| \longrightarrow \infty .
$$

${ }^{*}$ Corresponding author. Email addresses: fengxiaogao603@163.com (X. Feng), shjhuo@163.com (S. Huo) 
We denote the formal partial derivatives of $f$ by

$$
\partial f=f_{z}=\frac{\partial f}{\partial z}=\frac{1}{2}\left(\frac{\partial f}{\partial x}-i \frac{\partial f}{\partial y}\right) \quad \text { and } \quad \bar{\partial} f=f_{\bar{z}}=\frac{\partial f}{\partial \bar{z}}=\frac{1}{2}\left(\frac{\partial f}{\partial x}+i \frac{\partial f}{\partial y}\right),
$$

and use the notation

$$
|D f|=\left|f_{z}\right|+\left|f_{\bar{z}}\right| \text { and } J_{f}=\left|f_{z}\right|^{2}-\left|f_{\bar{z}}\right|^{2} .
$$

Here the value $|D f|$ is the operator norm for $D f$ and $J_{f}$ is the Jacobian of $f$.

A continuous function $E: \mathbb{R}^{n \times n} \longrightarrow \mathbb{R}$ is said to be quasiconvex if for every $f \in A+$ $C_{0}^{\infty}\left(\Omega, \mathbb{R}^{n}\right)$, we have

$$
\mathcal{E}[f]:=\int_{\Omega} E(D f) d x \geq \int_{\Omega} E(A) d x=E(A)|\Omega|,
$$

where $A$ stands for an arbitrary linear mapping (or its matrix) and $\Omega \subset \mathbb{R}^{n}$ is any bounded domain. In other words, one requires that compactly supported perturbations of linear maps do not decrease the value of the integral. This notion is very important in the calculus of variations [7]. Another notion is that of rank-one convexity, which requires just that $t \rightarrow E(A+t X)$ is convex for any fixed matrix $A$ and for any rank one matrix $X$. $E$ is rank-one concave (resp. quasiconcave) if $-E$ is rank-one convex (resp. quasiconvex). The most famous rank-one concave function in dimension two is the Burkholder functional defined for any $2 \times 2$ matrix $A$ by

$$
B_{P}(A)=\left[\frac{p}{2} \operatorname{det} A+\left(1-\frac{p}{2}\right)|A|^{2}\right]|A|^{p-2}, \quad p \geq 2 .
$$

where $|A|$ is the operator norm of $A$, see [3]. Morrey's work [8] implies that quasiconvexity implies rank-one convexity. For the dimension $n$ of $\mathbb{R}^{n}$ is bigger than 2 , Šverák's paper [10] showed that the converse is not true. However, for dimension $n=2$, [5] and [7] gave the evidence to the possibility for a different outcome. So in [1], the authors gave the following conjecture in the spirit of Morrey,

Conjecture 1.1. Rank-one convex functions $E: \mathbb{R}^{2 \times 2} \longrightarrow \mathbb{R}$ are quasiconvex.

For the $A=I d$, the authors of [1] showed that the Burkholder function is quasiconcave within quasiconformal perturbations of the identity. They showed that when $f: \Omega \rightarrow \Omega$ is a $k$-quasiconformal map of $\Omega$ onto itself with extending to the identity on the boundary, then

$$
\int_{\Omega} B_{p}(D f) d z=\int_{\Omega}\left(1-\frac{p|\mu(z)|}{1+|\mu(z)|}\right)\left(\left|f_{z}(z)\right|+\left|f_{\bar{z}}(z)\right|\right)^{p} d z \leq \int_{\Omega} B_{p}(I d) d z=|\Omega|
$$

where $2 \leq p \leq 1+\frac{1}{k}$.

In this paper, we first use the method learned from [1] to prove the following result: 
Theorem 1.1. Let $f: \mathbb{C} \rightarrow \mathbb{C}$ be the principal solution of a Beltrami equation

$$
f_{\bar{z}}(z)=\mu(z) f_{z}(z)
$$

for a given $\mu(z)$ with $|\mu(z)| \leq k \chi_{D}(z), 0 \leq k<1, D=\{z:|z|<1\}$ and it has the expansion

$$
f(z)=z+\frac{b_{1}}{z}+\frac{b_{2}}{z^{2}}+\cdots
$$

Then, for all $2 \leq p \leq 1+\frac{1}{k}$, we have

$\int_{D} B_{p}(D f)=\int_{D}\left(1-\frac{p|\mu(z)|}{1+|\mu(z)|}\right)\left(\left|f_{z}(z)\right|+\left|f_{\bar{z}}(z)\right|\right)^{p} d z \leq \int_{D} B_{2}\left(z+b_{1} \bar{z}\right) d z=\pi\left(1-\left|b_{1}\right|^{2}\right)$.

For the arbitrary bounded domain $\Omega$, we have the following result:

Theorem 1.2. Let $f: \Omega \rightarrow L(\Omega)$ be a $k$-quasiconformal map of the bounded $\Omega$ onto its affine image, extending continuously up to the boundary, where it coincides with the affine mapping $L$. Here $L(z)=z+\frac{b_{1}}{R^{2}} \bar{z}$ with $\left|b_{1}\right|<1$ is a linear mapping and

$$
R= \begin{cases}1, & \text { if } \operatorname{diam}(\Omega) \leq 2, \\ \frac{\operatorname{diam} \Omega}{2}, & \text { if } \operatorname{diam}(\Omega) \geq 2\end{cases}
$$

Then, for all $2 \leq p \leq 1+\frac{1}{k}$, we have

$$
\int_{\Omega} B_{p}(D f) d z \leq \int_{\Omega} B_{2}\left(z+\frac{b_{1}}{R^{2}} \bar{z}\right) d z=\left(1-\frac{\left|b_{1}\right|^{2}}{R^{4}}\right) .
$$

This paper is organized as follows. In Section 2, we will introduce some preliminary lemmas used in this paper. In Section 3 we give the proofs of Theorem 1.1 and Theorem 1.2. In the final section, we discuss some applications of the main theorems.

\section{Preliminary lemmas}

Let $\mathcal{M}(\Omega, \sigma)$ denote the class of complex valued $\sigma$-measurable functions on a measure space $(\Omega, \sigma)$. The following lemmas will be used in this note.

Lemma 2.1. (Interpolation Lemma for the disk [1]) Let $0<p_{0}, p_{1} \leq \infty$, and $\left\{\Phi_{\lambda}:|\lambda|<1\right\} \subset$ $\mathcal{M}(\Omega, \sigma)$ be an analytic and nonvanishing family with complex parameter $\lambda$ in the unit disc. Suppose

$$
M_{0}:=\left\|\Phi_{0}\right\|_{p_{0}}<\infty, \quad M_{1}:=\sup _{|\lambda|<1}\left\|\Phi_{\lambda}\right\|_{p_{1}}<\infty \quad \text { and } M_{r}:=\sup _{|\lambda|=r}|| \Phi_{\lambda} \|_{p_{r}}<\infty,
$$

where

$$
\frac{1}{p_{r}}=\frac{1-r}{1+r} \frac{1}{p_{0}}+\frac{2 r}{1+r} \frac{1}{p_{1}}
$$

Then, for every $0 \leq r<1$, we have

$$
M_{r} \leq M_{0}^{\frac{1-r}{1+r}} M_{1}^{\frac{2 r}{1+r}}<\infty
$$


Lemma 2.2. (Area Theorem [2]) Suppose that $f \in W_{\text {loc }}^{1,2}(\mathbb{C})$ is analytic outsider the disk $D(0, r)$ and has the expansion

$$
f(z)=z+\frac{b_{1}}{z}+\frac{b_{2}}{z}+\cdots, \text { for } \quad|z| \rightarrow \infty .
$$

Then

$$
\int_{D(0, r)} J(z, f) d z=\pi\left(r^{2}-\sum_{n=1}^{\infty} n\left|b_{n}\right|^{2} r^{-2 n}\right) .
$$

Lemma 2.3. ([4]) If $f$ is a $k$-quasiconformal mapping of a domain $\Omega \subset \mathbb{C}$ into $\mathbb{C}$, then for every $p \in\left[2,1+\frac{1}{k}\right)$,

$$
\frac{(1-(p-1) k)(1+k)^{p-1}}{\left(\sqrt{1-k^{2}}\right)^{p}}\left(\sqrt{J_{f}}\right)^{p} \leq B_{p}(D f) .
$$

\section{Proofs of Theorem 1.1 and Theorem 1.2}

First we will use holomorphic interpolation method and holomorphic motion to give the proof of Theorem 1.1 inspired by [1].

\section{Proof of Theorem 1.1}

We fix an exponent $2 \leq p \leq 1+\frac{1}{\|\mu\|_{\infty}}$ and look for holomorphic deformation of the given function $f$. To do this, we will construct an analytic family of Beltrami equations together with their principal solutions as the authors did in [1].

Let $F^{\lambda}(z)$ be the principal solutions of the following equations

$$
F_{\bar{z}}^{\lambda}=\mu_{\lambda}(z) F_{z}^{\lambda}, \quad \mu_{\lambda}(z)=\tau_{\lambda}(z) \frac{\mu(z)}{|\mu(z)|} .
$$

Here $\tau_{\lambda}(z)$ is an analytic function in $\lambda$ with

$$
\frac{\tau_{\lambda}(z)}{1+\tau_{\lambda}(z)}=p \frac{|\mu(z)|}{1+|\mu(z)|} \frac{\lambda}{1+\lambda}
$$

for $p=1+\frac{1}{\lambda_{0}}$. More explicitly,

$$
\tau_{\lambda}(z)=\frac{p \lambda|\mu(z)|}{(1+\lambda)(1+|\mu(z)|)-p \lambda|\mu(z)|} .
$$

It is easy to see that $\tau_{0}(z)=0$ and $F^{0}(z)=z$ as $\lambda=0$. There exists some complex number $\lambda=\lambda_{0}$ such that when $\lambda=\lambda_{0}$, we have $\tau_{\lambda_{0}}(z)=|\mu(z)|$, so that $F^{\lambda_{0}}(z)=f(z)$. Here and the following, we let $p=1+\frac{1}{\lambda_{0}}, p_{0}=\infty$ and $p_{2}=2$. We choose a nonvanishing analytic family $\left\{\Phi_{\lambda}\right\}_{|\lambda|<1}$ by

$$
\Phi_{\lambda}(z)=F_{z}^{\lambda}(z)\left(1+\tau_{\lambda}(z)\right)
$$


It is easy to see that for $\lambda=\lambda_{0}$,

$$
\left|\Phi_{\lambda_{0}}(z)\right|=(1+|\mu(z)|)\left|f_{z}\right|=\left|f_{z}\right|+\left|f_{\bar{z}}\right|=|D f| .
$$

We shall then apply Lemma 2.1 (the Interpolation Lemma) in the measure space $\mathcal{M}(D, \sigma)$ over the unit disk, where

$$
d \sigma(z, \lambda)=\frac{1}{\pi\left(1-\left|b_{1}^{\lambda}\right|^{2}\right)}\left(1-\frac{p|\mu(z)|}{1+|\mu(z)|}\right) d z
$$

and $b_{1}^{\lambda}$ is the coefficient of $\frac{1}{z}$ for the expansion of $F^{\lambda}(z)$. In particular, for $\lambda=\lambda_{0}$, we have

$$
d \sigma\left(z, \lambda_{0}\right)=\frac{1}{\pi\left(1-\left|b_{1}\right|^{2}\right)}\left(1-\frac{p|\mu(z)|}{1+|\mu(z)|}\right) d z
$$

where $b_{1}$ is the coefficient of $\frac{1}{z}$ for the expansion of $f$.

When $\lambda=0, F^{0}(z)=z$, hence $F_{z}^{0} \equiv 1, \Phi_{0} \equiv 1$ and $M_{0}=\left\|\Phi_{0}\right\|_{\infty}=1$. In the follows we will estimate $M_{1}=\sup _{\lambda \in D}\left\|\Phi_{\lambda}\right\|_{2} \leq 1$.

In view of Lemma 2.2

$$
\int_{D} J\left(z, F^{\lambda}\right) d z \leq \pi\left(1-\left|b_{1}^{\lambda}\right|^{2}\right),
$$

with equality if and only if $F^{\lambda}=z+\frac{e^{i \theta} b_{1}^{\lambda}}{z}$, for some $\theta \in(0,2 \pi)$, outside the unit disk.

We find that

$$
\begin{aligned}
& J\left(z, F^{\lambda}\right)=\left|F_{z}^{\lambda}(z)\right|^{2}\left(1-\left|\mu_{\lambda}(z)\right|^{2}\right)=\left|\Phi_{\lambda}(z)\right|^{2}\left(1-2 \operatorname{Re} \frac{\tau_{\lambda}(z)}{1+\tau_{\lambda}(z)}\right) \\
= & \left|\Phi_{\lambda}(z)\right|^{2}\left(1-p \frac{|\mu(z)|}{1+|\mu(z)|} \operatorname{Re} \frac{2 \lambda}{1+\lambda}\right) \geq\left|\Phi_{\lambda}(z)\right|^{2}\left(1-p \frac{|\mu(z)|}{1+|\mu(z)|}\right) .
\end{aligned}
$$

Hence

$$
\left|\Phi_{\lambda}(z)\right|^{2} d \sigma(z, \lambda) \leq \frac{1}{\pi\left(1-\left|b_{1}^{\lambda}\right|^{2}\right)} J\left(z, F^{\lambda}\right) d z
$$

therefore

$$
M_{1}=\sup _{|\lambda|<1} \int_{D}\left|\Phi_{\lambda}(z)\right|^{2} d \sigma(z, \lambda) \leq \sup _{|\lambda|<1} \frac{1}{\pi\left(1-\left|b_{1}^{\lambda}\right|^{2}\right)} \int_{D} J\left(z, F^{\lambda}\right) d z \leq 1 .
$$

For every $0 \leq r<1$, in view of the Interpolation Lemma, we have

$$
M_{r}=\sup _{|\lambda|=r}\left\{\left\|\Phi_{\lambda}\right\|_{\frac{1+r}{r}}\right\} \leq M_{0}^{\frac{1-r}{1+r}} M_{1}^{\frac{2 r}{1+r}} \leq 1
$$

After the substitution $r=\frac{1}{P-1}=\lambda_{0}$, the inequality is immediate

$$
\int_{D}\left(1-\frac{p|\mu(z)|}{1+|\mu(z)|}\right)|D f|^{p}=\pi\left(1-\left|b_{1}\right|^{2}\right) \int_{D}\left|\Phi_{\lambda_{0}}\right|^{\frac{1+r}{r}} d \sigma\left(z, \lambda_{0}\right) \leq \pi\left(1-\left|b_{1}\right|^{2}\right)=\int_{D} B_{2}\left(z+b_{1} \bar{z}\right) d z .
$$


So the proof of Theorem 1.1 is complete.

\section{Proof of Theorem 1.2}

Since

$$
R= \begin{cases}1, & \text { if } \quad \operatorname{diam}(\Omega) \leq 2, \\ \frac{\operatorname{diam} \Omega}{2}, & \text { if } \quad \operatorname{diam}(\Omega) \geq 2,\end{cases}
$$

we get $D_{R} \supset \Omega$. In view of the proof of Theorem 1.1, we just let

$$
d \sigma(z, \lambda)=\frac{1}{\pi R^{2}\left(1-\frac{\left|b_{1}^{\lambda}\right|^{2}}{R^{4}}\right)}\left(1-\frac{p|\mu(z)|}{1+|\mu(z)|}\right) d z,
$$

and can obtain following

$$
\int_{D_{R}} B_{p}(D f) d z \leq \int_{D_{R}} B_{2}\left(z+\frac{b_{1}}{R^{2}} \bar{z}\right) d z=\left(1-\frac{\left|b_{1}\right|^{2}}{R^{4}}\right)\left|D_{R}\right| .
$$

Let

$$
\widetilde{f}(z)=\left\{\begin{array}{lll}
f(z), & \text { if } \quad z \in \Omega, \\
z+\frac{b_{1}}{R^{2}} \bar{z}, & \text { if } \quad z \in D_{R}-\Omega, \\
z+\frac{b_{1}}{R^{2}} \frac{1}{z}, & \text { if } & z \in \mathbb{C}-D_{R} .
\end{array}\right.
$$

By inequality (3.1), this yields

$$
\int_{D_{R}} B_{p}(D \widetilde{f}) \leq \int_{D_{R}} B_{2}\left(z+\frac{b_{1}}{R^{2}} \bar{z}\right)
$$

And since

$$
\int_{D_{R}-\Omega} B_{p}(D \widetilde{f})=\int_{D_{R}-\Omega} B_{2}\left(z+\frac{b_{1}}{R^{2}} \bar{z}\right),
$$

in view of (3.2) and (3.3), we get

$$
\int_{\Omega} B_{P}(D f) d z \leq \int_{\Omega} B_{2}\left(z+\frac{b_{1}}{d^{2}} \bar{z}\right) d z=|\Omega|\left(1-\frac{\left|b_{1}\right|^{2}}{R^{4}}\right) .
$$

So the proof of Theorem 1.2 is complete.

\section{Upper bounds of the $L^{p}$-integrals of $\sqrt{J_{f}}$ and $|D f|$}

In this section, as an application of Theorem 1.1 or Theorem 1.2, we obtain upper bounds of the $L^{p}$-integrals of $\sqrt{J_{f}}$ and $|D f|$.

Theorem 4.1. Let $f: \Omega \rightarrow L(\Omega)$ be a $k$-quasiconformal map of the bounded $\Omega$ onto its affine image, extending continuously up to the boundary, where it coincides with the affine mapping $L$. Here we assume that the mapping $L$ satisfies $L(z)=z+\frac{b_{1}}{R^{2}} \bar{z}$ with some $\left|b_{1}\right|<1$ and

$$
R= \begin{cases}1, & \text { if } \quad \operatorname{diam}(\Omega) \leq 2 \\ \frac{\operatorname{diam} \Omega}{2}, & \text { if } \quad \operatorname{diam}(\Omega) \geq 2\end{cases}
$$


Then, for all $2 \leq p \leq 1+\frac{1}{k}$, we have

$$
\int_{\Omega}\left(\sqrt{J_{f}}\right)^{p} d z \leq \frac{\left(\sqrt{1-k^{2}}\right)^{p}}{(1-(p-1) k)(1+k)^{p-1}} \cdot\left(1-\frac{\left|b_{1}\right|^{2}}{R^{4}}\right)|\Omega| .
$$

Proof. By the Lemma 2.3, for every $2 \leq p \leq 1+\frac{1}{k}$, we may write

$$
\int_{\Omega}\left(\sqrt{J_{f}}\right)^{p} d z \leq \frac{\left(\sqrt{1-k^{2}}\right)^{p}}{(1-(p-1) k)(1+k)^{p-1}} \int_{\Omega} B_{p}(D f) d z .
$$

By Theorem 1.2 and (4.1), we have

$$
\int_{\Omega}\left(\sqrt{J_{f}}\right)^{p} d z \leq \frac{\left(\sqrt{1-k^{2}}\right)^{p}}{(1-(p-1) k)(1+k)^{p-1}} \cdot\left(1-\frac{\left|b_{1}\right|^{2}}{R^{4}}\right)|\Omega| .
$$

Corollary 4.1. Let $f: \Omega \rightarrow L(\Omega)$ be a $k$-quasiconformal map of the bounded $\Omega$ onto its affine image, extending continuously up to the boundary, where it coincides with the affine mapping $L$. Here $L(z)=z+\frac{b_{1}}{R^{2}} \bar{z}$ with $\left|b_{1}\right|<1$ is a linear mapping and

$$
R= \begin{cases}1, & \text { if } \quad \operatorname{diam}(\Omega) \leq 2 \\ \frac{\operatorname{diam} \Omega}{2}, & \text { if } \quad \operatorname{diam}(\Omega) \geq 2\end{cases}
$$

Then, for all $2 \leq p \leq 1+\frac{1}{k}$, we have

$$
\int_{\Omega}|D f|^{p} d z \leq \frac{1+k}{1-(p-1) k} \cdot\left(1-\frac{\left|b_{1}\right|^{2}}{R^{4}}\right)|\Omega|
$$

Proof. By the equality

$$
\left(\sqrt{J_{f}}\right)^{p}=\left(\left|f_{z}\right|+\left|f_{\bar{z}}\right|\right)^{p}\left(\frac{\left|f_{z}\right|-\left|f_{\bar{z}}\right|}{\left|f_{z}\right|+\left|f_{\bar{z}}\right|}\right)^{\frac{p}{2}}
$$

we get from Theorem 4.1 that

$$
\left(\frac{1-k}{1+k}\right)^{\frac{p}{2}} \int_{\Omega}|D f|^{p} d z \leq \int_{\Omega}\left(\sqrt{J_{f}}\right)^{p} d z \leq \frac{\left(\sqrt{1-k^{2}}\right)^{p}}{(1-(p-1) k)(1+k)^{p-1}} \cdot\left(1-\frac{\left|b_{1}\right|^{2}}{R^{4}}\right) \cdot|\Omega| .
$$

Hence,

$$
\int_{\Omega}|D f|^{p} d z \leq \frac{1+k}{1-(p-1) k} \cdot\left(1-\frac{\left|b_{1}\right|^{2}}{R^{4}}\right) \cdot|\Omega| .
$$

The claim of Corollary 4.2 follows. 


\section{Acknowledgments}

The authors would like thank the referees for valuable suggestions. This work was supported by NSFC (Grant No.11401432) and the research startup foundation of China West Normal University (Grant No. 13D017).

\section{References}

[1] K. Astala, T. Iwaniec, I. Prause, E. Saksman. Burkholder integral, Morrey's problem and quasiconformal mappings. J. Amer. Math. Soc., 2012, 25: 507-531.

[2] K. Astala, T. Iwaniec, G.J. Martin. Elliptic partial differential equations and quasiconformal mappings in the plane. Princeton Mathematical Series, Princeton University Press, Princeton, 2009.

[3] D.L. Burkholder. Sharp inequalities for martingales and stochastic integrals. Colloque Paul Lévy sur les Processus Stochastiques, 75-94, 1988.

[4] X. Chen, T. Qian. A sharp lower bound of Burkholder's functional for $K$-quasiconformal mappings and its applications. Mona. Math., 2014.

[5] D. Faraco, L. Székelyhidi. Tartar's conjecture and localization of the quasiconvex hull in $\mathbb{R}^{2 \times 2}$. Acta. Math., 2008, 200: 279-305.

[6] P. Pedregal, V. Šverák. A note on quasiconvexity and Rank-one convexity for $2 \times 2$ matrices. J. Convex Anal., 1998, 5: 107-117.

[7] S. Müller. Quasi-convexity and lower semicontinuity of multiple integrals. Internat. Math. Res., 1999, 1087-1095.

[8] C.B. Morrey. Rank-one convexity implies quasiconvexity on diagonal matrices. Pacific J. Math.,1952, 2: 25-53.

[9] T. Iwaniec. Extremal inequalities in Sobolev spaces and quasiconformal mappings. Z. Anal. Anwendungen, 1982, 1: 1-16.

[10] V. Šverák. Rank-one convexity does not imply quasiconvexity. Proc. Roy. Edinburgh Sect.A, 1992, 120: 185-189. 